Body Shapes and Fit Analysis of ASTM Size Standards

for Overweight and Obese Men in the United States

\author{
Eonyou Shin, Virginia Tech \\ Elahe Saeidi, Oregon State University
}

Keywords: apparel fit, sizing, overweight and obese, body shape

\title{
Background and Significances
}

Obesity and being overweight has been increased that $30 \%$ of Americans are classified as overweight and with $36.5 \%$ of them considered obese (cdc.gov). In a study that used 3D body scanner to categorize men's body shapes (Shin et al., 2011), the researchers identified four body shapes among 3,686 male participants from SizeUSA data (i.e., slim shape, heavy shape, slant inverted triangle shape, and short round top shape). However, because the body shape categorization by Shin et al. (2011)'s study included the broader age range (i.e., 18-66+) and underweight men (i.e., BMI of 18.5 or less), there is a need to focus on overweight and obese men's body shape categorization. Although researchers conducted studies on women's body shape categorizations (e.g., Song \& Ashdown, 2011), it is critical to classify men's body shape in order to identify how well the current ASTM sizing systems would satisfy the overweight and obese men in the United States. Thus, the purpose of this study is to explore body shapes among overweight and obese men and examine the current ASTM sizing standards related to the categorized body shapes. Three research questions are as follows:

RQ1. What are body shapes among the overweight and obese men in the US?

RQ2. How well the body shapes fit to the current ASTM sizing standards?

RQ3. How fit problems differ across the body shapes based on the current ASTM sizing standards?

\section{Methods}

Men with BMI of 25 or over and between 18 and 55 years from the SizeUSA data (1818 useable data) was used in this study. Subjects were aged 18-25 (399, 21.9\%), 26-35 (460, 25.3\%), 36-45 $(535,29.4 \%)$, and 46-55 (424, 23.3\%). Over half of them $(1,125,61.9 \%)$ were overweight (BMI of 25 to 30 ) while $38.1 \%$ of them (693) were obese. Following previous studies on body shape categorization using the SizeUSA data (e.g., Song \& Ashdown, 2011), the additional data in the ImageTwin (TC2-19) software were extracted (i.e., widths and depths).

In the body shape categorization process, we followed previous study (Song \& Ashdown, 2011) to get the side seam and depth measurements. In an initial stage, a total number of 96 body measurements (i.e., 55 raw measurements and 41 drop values) were included in a bivariate correlation analysis. After deleting medium to high correlations with the weight, 20 measurements (i.e., 5 raw measurements and 15 drop values) were included in a principle component (PC) analysis. To reduce unnecessary measurements, several PC analyses were performed. According to the PC analyses, four PCs were identified with eigenvalues of 1.0 and 
greater and $77.8 \%$ of the variation among 8 variables: $\mathrm{PC} 1$ - Shoulder to bust front silhouette (i.e., Bust width, front shoulder width), PC2-Buttocks prominence (i.e., back depth: hip to waist, buttocks angle), PC3 — Bust to hip front silhouette (i.e., width: bust to waist, width: hip to waist), and PC4-Abdomen prominence (i.e., front arc: top hip to waist, front arc: hip to waist). Then, two cluster analyses, hierarchical and K-mean cluster analyses were used to specify the ideal numbers of body shapes for the overweight and obese men in the United States. To compare the sizing system with each body shape group, we used both ASTM sizing standards for men (D8077/8077M-16) (ASTM International, 2012) and big men (D8077/8077M-16) (ASTM International, 2016). Based on a formula for fit tolerance (Simmons et al, 2004), an appropriate size for each of five body parts (i.e., back shoulder length, four girth measurements on bust, waist, top hip and hip) was determined for each sizing standard. Values of ideal sizes on bust, waist, top hip, and hip were calculated. Fit analysis was performed for tops and bottoms based on the sizing charts in each sizing system. For tops, a perfect fit, "0", was recorded when there are no differences in sizes between bust and waist while a bad fit, " 1 ", was recorded for differences in sizes. For bottoms, "0" as a perfect fit was recorded when sizes in all the three areas (i.e., waist, top hip and hip) were identical while "1" was recorded as a bad fit when there were differences in sizes. For both tops and bottoms, "2" was recorded if one did not fall into the sizing system. Using a cross tabulation, associations between fit analysis for tops and bottoms and the body shape groups were examined to answer RQ2. To answer RQ3, an analysis of variance (ANOVA) and LSD pairwise comparison were performed to identify differences in mean values of size drops between two body parts across the different body shape groups.

\section{Results}

To answer RQ1, the three clusters (i.e., body shapes) were identified among the overweight and obese men. To determine the name of the three body shape groups, front and side silhouettes were visually examined in 3D virtual body model created in CLO software using mean values of the 8 measurements for each body shape group: Cluster 1 (BS1: Rectangle-curvy shape, $n=401$ 22.1\%), cluster 2 (BS2: Bottom hourglass-hip tilt shape, $\mathrm{n}=743,40.9 \%$ ), and cluster 3 (BS3: Top hourglass-straight shape, $\mathrm{n}=674,37.1 \%)$.

According to the crosstab analysis (RQ2), men with BS1 (87, 21.7\%) and BS3 (178, $26.4 \%)$ tend to find a perfect fit with tops more easily than BS2 $(125,18.6 \%)$ in the men size. About $80 \%$ of men with BS2 (597) would have fit problems with tops in the men size. In the big men size, men with BS1 $(81,20.2 \%)$ would be more likely to find a perfect fit with tops than other two groups (BS2: 77, 10.4\%, BS3: 71, 10.5\%). For bottoms, men with BS1 (8, 2.0\%) would have more difficulty to find a perfect fit in the men size than those with BS2 $(63,8.5 \%)$ and BS3 $(71,10.5 \%)$. However, this sizing standard would give men the most fit problems with bottoms for all the three groups (BS1: 346, 86.3\%, BS2: 644, 86.7\%, BS3: 565, 83.8\%). The big men sizing system tends to give the least perfect fit with bottoms to men with BS1 $(8,2.0 \%)$, BS2 $(5, .7 \%)$, and BS3 $(11,1.6 \%)$. Men with BS1 $(121,30.2 \%)$ would have most fit problems with bottoms for the big men size.

Page 2 of 3

(c) 2020 The author(s). Published under a Creative Commons Attribution License (https://creativecommons.org/licenses/by/4.0/), which permits unrestricted use, distribution, and reproduction in any medium, provided the original work is properly cited.

ITAA Proceedings, \#77 - https: / /itaaonline.org 
For RQ3, for tops in both men and big men sizes, men with BS3 have .4 size looser fit in their waist areas while men in the other two body shapes would have 1.1-1.5 sizes tighter fit in their waist areas when they go with the size based on their bust sizes. For bottoms in the men size, all the three body shapes would have looser fit on top hip (.5-.6 size) and hip (1.6-2.3 sizes) areas. In the big men size, men with BS1 would have tighter fit on top hip (1.5 sizes) but looser fit on hip (.7 size). For men with BS2 and BS3, they would have fit problems with bottoms due to tighter top hip (2.2-2.3 sizes) and hip (.4-1.2 sizes) areas in the big men size.

\section{Conclusions and Implications}

Three body shapes were categorized for the overweight and obese men in the United States: Rectangle-curvy shape, bottom hourglass-hip tilt shape, and top hourglass-straight shape. Result of this study support discrepancy between the body shapes and the current ASTM sizing standards. Overweight and obese men would experience more fit problems with bottoms than that with tops in both sizing standards (i.e., the men and the big men sizes). This study provides practical implications for apparel product developer in the United States that the sizing systems need to be updated to give better fit and accommodate different body shapes in the overweight and obese men. Further, this study contributes literature for body shape and fit analysis studies for the overweight and obese male population in the United States.

\section{References}

ASTM International. (2012). D8077/8077M-16, Standard tables of body measurements for mature men, ages 35 and older, Sizes thirty-four to fifty-two (34 to 52) short, regular, and tall Retrieved from: https://compass-astm-org.

ASTM International. (2016). D8077/8077M-16, Standard tables of body measurements for mature big men type, size range 46-64. Retrieved from: https://compass-astm-org.

Shin, S. H., Istook, C. L., \& Lee, J. H. (2011). Various men's body shapes and drops for developing menswear sizing systems in the United States, Journal of the Korean Society of Clothing and Textiles, 35(12), 1454-1465.

Simmons, K., Istook, C.L. \& Devarajan, P. (2004). Female figure identification technique (FFIT) for apparel part I: Describing female shapes. Journal of Textile and Apparel, Technology and Management, 4(1), 1-16.

Song, H. K., \& Ashdown, S. P. (2011). Categorization of lower body shapes for adult females based on multiple view analysis. Textile Research Journal, 81(9), 914-931.

Page 3 of 3

(c) 2020 The author(s). Published under a Creative Commons Attribution License

(https://creativecommons.org/licenses/by/4.0/), which permits unrestricted use, distribution, and reproduction in any medium, provided the original work is properly cited.

ITAA Proceedings, \#77 - https://itaaonline.org 